\title{
Nakajima-Zwanzig versus time-convolutionless master equation for the non-Markovian dynamics of a two-level system
}

\author{
Andrea Smirne ${ }^{1,2, *}$ and Bassano Vacchini ${ }^{1,2, \dagger}$ \\ ${ }^{1}$ Dipartimento di Fisica, Università degli Studi di Milano, Via Celoria 16, I-20133 Milano, Italy \\ ${ }^{2}$ Sezione di Milano, INFN, Via Celoria 16, I-20133 Milano, Italy
}

(Received 10 May 2010; published 18 August 2010)

\begin{abstract}
We consider the exact reduced dynamics of a two-level system coupled to a bosonic reservoir, further obtaining the exact time-convolutionless and Nakajima-Zwanzig non-Markovian equations of motion. The system considered includes the damped and undamped Jaynes-Cummings model. The result is obtained by exploiting an expression of quantum maps in terms of matrices and a simple relation between the time evolution map and the time-convolutionless generator as well as the Nakajima-Zwanzig memory kernel. This nonperturbative treatment shows that each operator contribution in Lindblad form appearing in the exact time-convolutionless master equation is multiplied by a different time-dependent function. Similarly, in the Nakajima-Zwanzig master equation each such contribution is convoluted with a different memory kernel. It appears that, depending on the state of the environment, the operator structures of the two sets of equations of motion can exhibit important differences.
\end{abstract}

DOI: 10.1103/PhysRevA.82.022110

PACS number(s): 03.65.Yz, 03.65.Ta, 42.50.Lc

\section{INTRODUCTION}

The study of open quantum systems is a wide research area of interest to various scientific communities, ranging from physicists to chemists and mathematicians. Its basic theoretical framework is well understood when a Markovian description can be applied [1,2], but despite important work [2,3], a lot remains to be clarified and understood when considering non-Markovian dynamics, which is often mandatory for a realistic approach, also coping with strong coupling between system and environment. Recently a lot of research work has been devoted to the subject [4-24]. On the one hand, it is not fully clear which is the most general operator structure of non-Markovian equations of motion, which do provide a well-defined time evolution and, in particular, preserve complete positivity. On the other hand, one would like to link, in a possibly intuitive way, operator structures giving a sensible dynamical evolution with microscopic information on the physics of the system of interest. For the Markovian case both these approaches, phenomenological and microscopic, are well understood and successfully applied. The result by Gorini et al. $[25,26]$ provides a robust framework in which to envisage a possible ansatz for the evolution equations, while the Markov approximation is often connected with weak coupling, thus making microscopic approaches more manageable. The non-Markovian case is much more subtle and involved, and results at the same level of generality are still not available. Moreover, building on the intuition gained for the Markovian case sometimes leads to inconsistencies and pitfalls. Indeed in the non-Markovian setting the formation of correlations between system and environment becomes of utmost importance, also because of the disappearance of a clear-cut separation of time scales between system and environment. The very assumption of a factorized state at some

\footnotetext{
*andrea.smirne@unimi.it

†bassano.vacchini@mi.infn.it
}

point in time, necessary to provide a well-defined dynamics in terms of the degrees of freedom of the system only, cannot always be reasonably assumed. To meet these challenges is a formidable task, whose success would lie in relaxing the too strong approximations involved in a Markovian assumption, still coming to a manageable theoretical description for the system of interest. To pursue this far-reaching goal it can be useful as an intermediate step to consider situations in which an exact characterization of a non-Markovian dynamics can be obtained.

In this article we discuss a realistic physical model, simple enough to be exactly treated in detail but already showing typical non-Markovian features. In particular, we not only derive the exact equations of motion, which provide the counterpart to the Lindblad structure of a Markovian model, but also obtain a clear-cut connection to the microscopic approach via the two standard Nakajima-Zwanzig and timeconvolutionless techniques. This work should help to provide a better understanding of typical non-Markovian features in simple but realistic models. We consider a two-level system coupled first to a single mode of the radiation field and later to a bath of harmonic oscillators, via a Jaynes-Cummings type of interaction. By exploiting the knowledge of the exact unitary evolution, and therefore of the reduced dynamics, as well as a suitable matrix representation of the dynamical maps, we can exhibit the exact time-convolutionless and Nakajima-Zwanzig equations of motion. It will appear that such equations are given by a sum of Lindblad terms, each multiplied or convoluted with a different time-dependent function. Moreover, the operator structure of these two sets of equations are shown to be generally different, also depending on the environmental state. These exact results, with a clear-cut connection to a microscopic perturbative derivation, should guide the development of a correct intuition for the approximate treatment of more involved non-Markovian systems.

The article is organized as follows. In Sec. II we derive the map giving the exact reduced dynamics for the JaynesCummings model, further introducing a simple representation 
of the map as a matrix with respect to a convenient basis of operators. In Sec. III we point out the simple relations connecting the time evolution map to the time-convolutionless, time-dependent generator and the Nakajima-Zwanzig memory kernel, respectively. These relationships are most easily expressed in matrix form. The corresponding matrices are evaluated for the reduced dynamics of our two-level system, further coming back to the operator expression of the master equations and giving the explicit result for a bath in the vacuum state. In Sec. IV we put forward a similar analysis for the damped two-level system, referring to previous results and comparing with the undamped case.

\section{JAYNES-CUMMINGS MODEL AND EXACT REDUCED DYNAMICS}

\section{A. Time evolution mapping}

We consider a two-level system coupled to a single mode of the radiation field according to the total Hamiltonian

$$
H=H_{S}+H_{E}+H_{I},
$$

where the system Hamiltonian is given by

$$
H_{S}=\omega_{0} \sigma_{+} \sigma_{-},
$$

with $\omega_{0}$ the transition frequency and $\sigma_{+}=|1\rangle\langle 0|$ and $\sigma_{-}=$ $|0\rangle\langle 1|$ the raising and lowering operators of the two-level system. The Hamiltonian for the single mode of the radiation field is given by

$$
H_{E}=\omega b^{\dagger} b,
$$

where the creation and annihilation operators $b^{\dagger}$ and $b$ obey the standard bosonic commutation relation. The coupling is in the Jaynes-Cummings form,

$$
H_{I}=g\left(\sigma_{+} \otimes b+\sigma_{-} \otimes b^{\dagger}\right),
$$

so that the considered model can describe, for example, the interaction between a two-level atom and a mode of the radiation field in electric dipole and rotating-wave approximation. Working in the interaction picture with respect to the free Hamiltonian $H_{S}+H_{E}$,

$$
H_{I}(t)=g\left(\sigma_{+} \otimes b e^{i \Delta t}+\sigma_{-} \otimes b^{\dagger} e^{-i \Delta t}\right),
$$

with

$$
\Delta=\omega_{0}-\omega
$$

the detuning between the system and the field mode, it is possible to obtain the exact dynamics generated by the total Hamiltonian (see, e.g., Ref. [27]) and, therefore, the reduced dynamics of the two-level system. We express the result exhibiting the unitary evolution operator which, in the basis $\{|1\rangle,|0\rangle\}$, is given by the following matrix, whose entries are operators in the Fock space of the radiation field

$$
U(t)=\left(\begin{array}{cc}
c(\hat{n}+1, t) & d(\hat{n}+1, t) b \\
-b^{\dagger} d^{\dagger}(\hat{n}+1, t) & c^{\dagger}(\hat{n}, t)
\end{array}\right),
$$

where the following operators have been introduced:

$$
\begin{aligned}
& c(\hat{n}, t)=e^{i \Delta t / 2}\left[\cos \left(\sqrt{\Delta^{2}+4 g^{2} \hat{n}} \frac{t}{2}\right)\right. \\
& \left.-i \Delta \frac{\sin \left(\sqrt{\Delta^{2}+4 g^{2} \hat{n}} \frac{t}{2}\right)}{\sqrt{\Delta^{2}+4 g^{2} \hat{n}}}\right] \text {, } \\
& d(\hat{n}, t)=-i e^{i \Delta t / 2} 2 g \frac{\sin \left(\sqrt{\Delta^{2}+4 g^{2} \hat{n}} \frac{t}{2}\right)}{\sqrt{\Delta^{2}+4 g^{2} \hat{n}}},
\end{aligned}
$$

with $\hat{n}=b^{\dagger} b$ the number operator. The unitarity of $U(t)$ is granted because of the easily verified relation

$$
c^{\dagger}(\hat{n}, t) c(\hat{n}, t)+\hat{n} d^{\dagger}(\hat{n}, t) d(\hat{n}, t)=1 .
$$

Given the unitary evolution of the whole bipartite system, one can obtain the reduced dynamics of the two-level atom simply by taking the partial trace with respect to the environmental degrees of freedom. If the initial state of the system and environment is factorized, the map giving the reduced dynamics is completely positive and takes the form

$$
\begin{aligned}
\rho(t) & =\operatorname{Tr}_{E}\left[U(t) \rho(0) \otimes \rho_{E} U^{\dagger}(t)\right] \\
& \equiv \Phi(t) \rho(0) .
\end{aligned}
$$

Taking $U(t)$ as in Eq. (7) and considering an environmental state commuting with the number operator, $\left[\rho_{E}, n\right]=0$, so that, in particular, both the vacuum and a thermal state can be dealt with, one comes to the following explicit expression for the action of the map $\Phi(t)$ :

$$
\begin{aligned}
\rho_{11}(t)= & \rho_{00}(0)[1-\alpha(t)]+\rho_{11}(0) \beta(t), \\
& \rho_{10}(t)=\rho_{10}(0) \gamma(t), \\
& \rho_{01}(t)=\rho_{01}(0) \gamma^{*}(t), \\
\rho_{00}(t)= & \rho_{00}(0) \alpha(t)+\rho_{11}(0)[1-\beta(t)] .
\end{aligned}
$$

The effect of the interaction with the bath is contained in the time-dependent coefficients $\alpha(t), \beta(t)$, and $\gamma(t)$, which are given by the following expectation values over the state of the environment $\rho_{E}$ :

$$
\begin{gathered}
\alpha(t)=\left\langle c^{\dagger}(\hat{n}, t) c(\hat{n}, t)\right\rangle_{E}, \\
\beta(t)=\left\langle c^{\dagger}(\hat{n}+1, t) c(\hat{n}+1, t)\right\rangle_{E}, \\
\gamma(t)=\langle c(\hat{n}, t) c(\hat{n}+1, t)\rangle_{E} .
\end{gathered}
$$

\section{B. Matrix representation}

Now that we have obtained the completely positive map $\Phi(t)$ giving the exact reduced time evolution of the considered two-level system, we shall express it for later convenience in matrix form with respect to a suitable basis of operators in the Hilbert space $\mathbb{C}^{2}$, following [28]. Indeed, having fixed a basis $\left\{X_{l}\right\}_{l=0,1,2,3}$ of operators in $\mathbb{C}^{2}$, orthonormal according to $\operatorname{Tr}_{S}\left[X_{k}^{\dagger} X_{l}\right]=\delta_{k l}$, a linear map $\Lambda$ can be expressed in this 
basis according to

$$
\Lambda \rho=\sum_{k l} L_{k l} \operatorname{Tr}_{S}\left[X_{l}^{\dagger} \rho\right] X_{k},
$$

where the matrix of coefficients $L_{k l}$ uniquely associated with the map is given by

$$
L_{k l}=\operatorname{Tr}_{S}\left[X_{k}^{\dagger} \Lambda\left(X_{l}\right)\right] .
$$

Here and in the following we use Greek or calligraphic letters to denote maps and Roman letters to indicate the corresponding matrix. The convenient choice of basis for our calculations, to later recast the relevant maps in operator form, is given by $\left\{X_{l}\right\}_{l=0, i} \equiv\left\{\frac{1}{\sqrt{2}} \mathbb{1}, \frac{1}{\sqrt{2}} \sigma_{i}\right\}$, where $\sigma_{i}$ denote the usual Pauli operators. This choice leads to the following expression for the matrix $F_{k l}(t)$ associated with the time evolution map $\Phi(t)$ :

$$
F(t)=\left(\begin{array}{cccc}
1 & 0 & 0 & 0 \\
0 & \gamma_{R}(t) & \gamma_{I}(t) & 0 \\
0 & -\gamma_{I}(t) & \gamma_{R}(t) & 0 \\
\beta(t)-\alpha(t) & 0 & 0 & \beta(t)+\alpha(t)-1
\end{array}\right),
$$

where the coefficients defined in Eq. (13) appear, and we denote by $R$ and $I$ the real and imaginary parts of a given function:

$$
\gamma=\gamma_{R}+i \gamma_{I}
$$

This strategy of associating matrices with maps, already pursued in [28], which transforms the composition of maps in matrix multiplication, will turn out to be very convenient for obtaining the expressions of exact equations of motion leading to the dynamics described by Eq. (12).

\section{EXACT NAKAJIMA-ZWANZIG AND TIME-CONVOLUTIONLESS MASTER EQUATIONS}

\section{A. General expression in terms of the time evolution map}

With the aid of the knowledge of the exact time evolution, and using the representation of maps in terms of matrices, we now explicitly obtain two kinds of exact equations of motion

$$
K_{\mathrm{TCL}}(t)=\left(\begin{array}{c}
0 \\
0 \\
0 \\
\frac{[1-2 \beta(t)] \dot{\alpha}(t)-[1-2 \alpha(t)] \dot{\beta}(t)}{\beta(t)+\alpha(t)-1}
\end{array}\right.
$$

and the expression is well defined provided the determinant

$$
\operatorname{det} F(t)=|\gamma(t)|^{2}[\alpha(t)+\beta(t)-1]
$$

is different from 0 . for the reduced system's dynamics. We first consider a master equation in differential form with a generator local in time, that is, the time-convolutionless master equation. Assuming the existence of such a generator $\mathcal{K}_{\mathrm{TCL}}(t)$, it should obey the equation

$$
\dot{\rho}(t)=\mathcal{K}_{\mathrm{TCL}}(t) \rho(t),
$$

which, due to $\rho(t)=\Phi(t) \rho(0)$, is satisfied upon identifying

$$
\mathcal{K}_{\mathrm{TCL}}(t)=\dot{\Phi}(t) \Phi^{-1}(t)
$$

or, in terms of matrices,

$$
K_{\mathrm{TCL}}(t)=\dot{F}(t) F^{-1}(t),
$$

holding, provided the inverse does exist.

On a similar footing one can consider a master equation in integrodifferential form, given by a suitable memory kernel, corresponding to the Nakajima-Zwanzig master equation. In this case the memory kernel $\mathcal{K}_{\mathrm{NZ}}(t)$ should obey the convolution equation

$$
\dot{\rho}(t)=\left(\mathcal{K}_{\mathrm{NZ}} \circ \rho\right)(t),
$$

so that in view of Eq. (11), one has the relation

$$
\widehat{\mathcal{K}}_{\mathrm{NZ}}(u)=u \mathbb{1}-\hat{\Phi}^{-1}(u),
$$

where the hat denotes the Laplace transform, and therefore in matrix representation,

$$
\hat{K}_{\mathrm{NZ}}(u)=u \mathbb{1}-\hat{F}^{-1}(u) .
$$

It immediately appears that, given the time evolution map from the relationships Eqs. (19) and (22), one can directly obtain the generator of the master equation in time-convolutionless form or the memory kernel for the Nakajima-Zwanzig form, respectively, without resorting to the evaluation of the whole perturbative series. Obviously, given the exact time evolution, one does not need the equations of motion. Nevertheless, an exact comprehensive study as is feasible in this case allows us, as we shall see, to point out general features, serving as a guide for phenomenological or approximate treatments.

Starting from Eqs. (16) and (19), one obtains for the model of interest

$$
\left.\begin{array}{ccc}
0 & 0 & 0 \\
\operatorname{Re}\left[\frac{\dot{\gamma}(t)}{\gamma(t)}\right] & \operatorname{Im}\left[\frac{\dot{\gamma}(t)}{\gamma(t)}\right] & 0 \\
-\operatorname{Im}\left[\frac{\dot{\gamma}(t)}{\gamma(t)}\right] & \operatorname{Re}\left[\frac{\dot{\gamma}(t)}{\gamma(t)}\right] & 0 \\
0 & 0 & \frac{\dot{\beta}(t)+\dot{\alpha}(t)}{\beta(t)+\alpha(t)-1}
\end{array}\right),
$$

On a similar footing one can consider the Laplace transform of Eq. (16), given by the matrix $\hat{F}(u)$ with determinant

$$
\operatorname{det} \hat{F}(u)=\left[\hat{\gamma}_{R}^{2}(u)+\hat{\gamma}_{I}^{2}(u)\right]\left[\frac{\hat{\alpha}(u)+\hat{\beta}(u)}{u}-\frac{1}{u^{2}}\right] \text {, }
$$

and using Eq. (22) one further obtains 


$$
\hat{K}_{\mathrm{NZ}}(u)=\left(\begin{array}{cccc}
0 & 0 & 0 & 0 \\
0 & u-\frac{\hat{\gamma}_{R}(u)}{\hat{\gamma}_{R^{2}}(u)+\hat{\gamma}_{I}^{2}(u)} & \frac{\hat{\gamma}_{I}(u)}{\hat{\gamma}^{2}(u)+\hat{\gamma}_{I}^{2}(u)} & 0 \\
0 & -\frac{\hat{\gamma}_{I}(u)}{\hat{\gamma}_{R}^{2}(u)+\hat{\gamma}_{I}^{2}(u)} & u-\frac{\hat{\gamma}_{R}(u)}{\hat{\gamma}_{R}^{2}(u)+\hat{\gamma}_{I}^{2}(u)} & 0 \\
\frac{u^{2}[\hat{\alpha}(u)-\hat{\beta}(u)]}{1-u[\hat{\alpha}(u)+\hat{\beta}(u)]} & 0 & 0 & \frac{2 u-u^{2}[\hat{\alpha}(u)+\hat{\beta}(u)]}{1-u[\hat{\alpha}(u)+\hat{\beta}(u)]}
\end{array}\right),
$$

which, upon inverse Laplace transform, provides the exact Nakajima-Zwanzig integral kernel. As it appears, working with the matrix representation has proved very convenient for easily obtaining the maps fixing the differential and integrodifferential equations of motion for the model, given by Eqs. (17) and (20), respectively, in terms of the completely positive time evolution map, Eq. (12).

\section{B. Operator expression}

We now recast the obtained maps, providing a time-local generator and memory kernel, in operator form, for better comparison with previous work and appreciation of the difference in the obtained expressions. This is easily done by observing that a matrix of the form

$$
A=\left(\begin{array}{cccc}
0 & 0 & 0 & 0 \\
0 & E_{r} & E_{i} & 0 \\
0 & -E_{i} & E_{r} & 0 \\
X & 0 & 0 & Y
\end{array}\right)
$$

in the basis $\left\{\frac{1}{\sqrt{2}} \mathbb{1}, \frac{1}{\sqrt{2}} \sigma_{i}\right\}$ corresponds in operator form to the map

$$
\begin{aligned}
\mathcal{A} \rho= & i E_{i}\left[\sigma_{+} \sigma_{-}, \rho\right]+\frac{1}{2}(X-Y)\left[\sigma_{+} \rho \sigma_{-}-\frac{1}{2}\left\{\sigma_{-} \sigma_{+}, \rho\right\}\right] \\
& -\frac{1}{2}(X+Y)\left[\sigma_{-} \rho \sigma_{+}-\frac{1}{2}\left\{\sigma_{+} \sigma_{-}, \rho\right\}\right] \\
& +\frac{1}{4}\left(Y-2 E_{r}\right)\left[\sigma_{z} \rho \sigma_{z}-\rho\right]
\end{aligned}
$$

whose last term can be written in alternative ways according to the identities

$$
\begin{aligned}
\sigma_{z} \rho \sigma_{z}-\rho & =4\left[\sigma_{+} \sigma_{-} \rho \sigma_{+} \sigma_{-}-\frac{1}{2}\left\{\sigma_{+} \sigma_{-}, \rho\right\}\right] \\
& =4\left[\sigma_{-} \sigma_{+} \rho \sigma_{-} \sigma_{+}-\frac{1}{2}\left\{\sigma_{-} \sigma_{+}, \rho\right\}\right] .
\end{aligned}
$$

Exploiting this result one obtains the exact timeconvolutionless master equation describing the reduced dynamics of a two-level atom coupled, according to the Jaynes-Cummings model, to a single mode of the radiation field, which is of the form Eq. (17), with $\mathcal{K}_{\mathrm{TCL}}(t)$ given by

$$
\begin{aligned}
& \mathcal{K}_{\mathrm{TCL}}(t) \rho=i \operatorname{Im}\left[\frac{\dot{\gamma}(t)}{\gamma(t)}\right]\left[\sigma_{+} \sigma_{-}, \rho\right] \\
& +\frac{[\alpha(t)-1] \dot{\beta}(t)-\beta(t) \dot{\alpha}(t)}{\beta(t)+\alpha(t)-1}\left[\sigma_{+} \rho \sigma_{-}-\frac{1}{2}\left\{\sigma_{-} \sigma_{+}, \rho\right\}\right]
\end{aligned}
$$

$$
\begin{aligned}
& +\frac{[\beta(t)-1] \dot{\alpha}(t)-\alpha(t) \dot{\beta}(t)}{\beta(t)+\alpha(t)-1}\left[\sigma_{-} \rho \sigma_{+}-\frac{1}{2}\left\{\sigma_{+} \sigma_{-}, \rho\right\}\right] \\
& +\frac{1}{4}\left\{\frac{\dot{\beta}(t)+\dot{\alpha}(t)}{\beta(t)+\alpha(t)-1}-2 \operatorname{Re}\left[\frac{\dot{\gamma}(t)}{\gamma(t)}\right]\right\}\left[\sigma_{z} \rho \sigma_{z}-\rho\right] .
\end{aligned}
$$

In a similar way one has for the Laplace transform of the memory kernel $\mathcal{K}_{\mathrm{NZ}}(t)$, appearing in the exact NakajimaZwanzig master equation, Eq. (20), the expression

$$
\begin{aligned}
& \widehat{\mathcal{K}}_{\mathrm{NZ}}(u) \rho=i \frac{\hat{\gamma}_{I}(u)}{\hat{\gamma}_{R}^{2}(u)+\hat{\gamma}_{I}^{2}(u)}\left[\sigma_{+} \sigma_{-}, \rho\right] \\
& +\frac{u[u \hat{\alpha}(u)-1]}{1-u[\hat{\alpha}(u)+\hat{\beta}(u)]}\left[\sigma_{+} \rho \sigma_{-}-\frac{1}{2}\left\{\sigma_{-} \sigma_{+}, \rho\right\}\right] \\
& \quad+\frac{u[u \hat{\beta}(u)-1]}{1-u[\hat{\alpha}(u)+\hat{\beta}(u)]}\left[\sigma_{-} \rho \sigma_{+}-\frac{1}{2}\left\{\sigma_{+} \sigma_{-}, \rho\right\}\right] \\
& \quad+\frac{1}{4}\left\{\frac{u^{2}[\hat{\alpha}(u)+\hat{\beta}(u)]}{1-u[\hat{\alpha}(u)+\hat{\beta}(u)]}+2 \frac{\hat{\gamma}_{R}(u)}{\hat{\gamma}_{R}^{2}(u)+\hat{\gamma}_{I}^{2}(u)}\right\} \\
& \quad \times\left[\sigma_{z} \rho \sigma_{z}-\rho\right] .
\end{aligned}
$$

Despite being exact, these expressions are quite cumbersome, since the functions given in Eq. (13) — which, together with their Laplace transform, determine the structure of these operators - depend on the specific expression of the environmental state. It is therefore convenient to consider a specific choice, allowing for a more detailed evaluation.

\section{The vacuum case}

If the radiation field is in the vacuum state, the functions given in Eq. (13) simplify considerably, since $\alpha(t) \rightarrow 1$, while $\beta(t)$ becomes a function of $\gamma(t)$ according to $\beta(t) \rightarrow|\gamma(t)|^{2}$. The function $\gamma(t)$ for the vacuum case is given by the expression

$$
G^{1}(t)=e^{i \Delta t / 2}\left[\cos \left(\frac{\Omega_{1} t}{2}\right)-i \frac{\Delta}{\Omega_{1}} \sin \left(\frac{\Omega_{1} t}{2}\right)\right],
$$

where the superscript recalls that we have a single mode of the radiation field, while $\Omega_{1}=\sqrt{\Delta^{2}+4 g^{2}}$. These results for the vacuum case greatly simplify the expression of the obtained master equations and, inserted in Eq. (24), show that the timeconvolutionless master equation off-resonance is always well defined. 
Indeed the time-convolutionless master equation for the vacuum case simply reads

$$
\begin{aligned}
\mathcal{K}_{\mathrm{TCL}}^{\mathrm{Vac}}(t) \rho= & +i \operatorname{Im}\left[\frac{\dot{G}^{1}(t)}{G^{1}(t)}\right]\left[\sigma_{+} \sigma_{-}, \rho\right] \\
& -2 \operatorname{Re}\left[\frac{\dot{G}^{1}(t)}{G^{1}(t)}\right]\left[\sigma_{-} \rho \sigma_{+}-\frac{1}{2}\left\{\sigma_{+} \sigma_{-}, \rho\right\}\right],
\end{aligned}
$$

corresponding, as expected, to a Lindblad structure with time-dependent coefficients. Exploiting Eq. (32), one has the explicit expression

$$
\begin{aligned}
\mathcal{K}_{\mathrm{TCL}}^{\mathrm{Vac}}(t) \rho= & -i g^{2} \Delta \frac{1-\cos \left(\Omega_{1} t\right)}{\Omega_{1}}\left[\cos ^{2}\left(\frac{\Omega_{1} t}{2}\right)\right. \\
& \left.+\frac{\Delta^{2}}{\Omega_{1}^{2}} \sin ^{2}\left(\frac{\Omega_{1} t}{2}\right)\right]^{-1}\left[\sigma_{+} \sigma_{-}, \rho\right]+2 g^{2} \frac{\sin \left(\Omega_{1} t\right)}{\Omega_{1}} \\
& \times\left[\cos ^{2}\left(\frac{\Omega_{1} t}{2}\right)+\frac{\Delta^{2}}{\Omega_{1}^{2}} \sin ^{2}\left(\frac{\Omega_{1} t}{2}\right)\right]^{-1} \\
& \times\left[\sigma_{-} \rho \sigma_{+}-\frac{1}{2}\left\{\sigma_{+} \sigma_{-}, \rho\right\}\right]
\end{aligned}
$$

where, in particular, one directly sees that the coefficient in front of the dissipative term on the right-hand side of Eq. (34) periodically takes on negative values, so that it describes a truly non-Markovian behavior and a Markovian approximation is not justified even for long times.

The choice of the vacuum as bath state brings in important simplifications also for the expression of the NakajimaZwanzig memory kernel, whose Laplace transform reads

$$
\begin{aligned}
& \widehat{\mathcal{K}}_{\mathrm{NZ}}^{\mathrm{Vac}}(u) \rho \widehat{G_{I}^{1}}(u) \\
&=+i \frac{{\widehat{G_{R}^{1}}}^{2}(u)+{\widehat{G_{I}^{1}}}^{2}(u)}{\left.\sigma_{+} \sigma_{-}, \rho\right]} \\
&+\left[\frac{1-u \hat{z}^{1}(u)}{\hat{z}^{1}(u)}\right]\left[\sigma_{-} \rho \sigma_{+}-\frac{1}{2}\left\{\sigma_{+} \sigma_{-}, \rho\right\}\right] \\
&-\frac{1}{4}\left[\frac{1-u \hat{z}^{1}(u)}{\hat{z}^{1}(u)}+2\left(u-{\widehat{G_{R}^{1}}}^{2}(u)\right.\right. \\
& \quad \times\left[\sigma_{z} \rho \sigma_{z}-\rho\right],
\end{aligned}
$$

where

$$
z^{1}(t)=\left|G^{1}(t)\right|^{2} .
$$

Taking into account the explicit result Eq. (32), one obtains, after some calculations, the following compact expression for the memory kernel:

$$
\begin{aligned}
\mathcal{K}_{\mathrm{NZ}}^{\mathrm{Vac}} & (\tau) \rho \\
= & -i g^{2} \sin (\Delta \tau)\left[\sigma_{+} \sigma_{-}, \rho\right]+2 g^{2} \cos \left(\sqrt{\Delta^{2}+2 g^{2}} \tau\right) \\
& \times\left[\sigma_{-} \rho \sigma_{+}-\frac{1}{2}\left\{\sigma_{+} \sigma_{-}, \rho\right\}\right]-\frac{1}{2} g^{2}\left[\cos \left(\sqrt{\Delta^{2}+2 g^{2}} \tau\right)\right. \\
& -\cos (\Delta \tau)]\left[\sigma_{z} \rho \sigma_{z}-\rho\right],
\end{aligned}
$$

which is always well defined, even on-resonance. These results already allow for a few important remarks. We first notice that the different operator contributions in Lindblad form appearing in the various time-local and integral kernels are multiplied by different time-dependent functions. This lesson, already learned in other models [15,20,29,30], tells us that as a general rule non-Markovian dynamics cannot be obtained from Markovian expressions by simply taking the convolution with a single integral kernel or multiplying by a single time-dependent function. This fact has often been overlooked when seeking non-Markovian integrodifferential master equations [31-34], sometimes leading to unphysical behaviors. More than this, for the same model different sets of non-Markovian equations of motion can have different operator structures, as it appears upon comparison of, for example, the time-convolutionless and the Nakajima-Zwanzig results for the vacuum Eqs. (33) and (37). This fact was already noticed in [20], but the present analysis shows that this asymmetry depends on the choice of environmental state. For the present model it only appears in connection with the vacuum state. Indeed, while the disappearance of the term corresponding to excitation of the two-level system is obvious on physical grounds, when considering the vacuum as the bath state, the vanishing of the coefficient in front of the dephasing term $\sigma_{z} \rho \sigma_{z}-\rho$ is a peculiar feature of the time-convolutionless master equation.

\section{DAMPED TWO-LEVEL SYSTEM}

\section{A. Exact master equations for the bath in the vacuum state}

The technique used in Sec. III to obtain the timeconvolutionless and Nakajima-Zwanzig equations of motion for a model whose evolution is known, by exploiting the representation of maps in terms of matrices, is applicable for a detailed study of simple models. We exploit it now for a variant of the model considered in Sec. II, in which the environmental Hamiltonian is a collection of harmonic oscillators,

$$
H_{E}=\sum_{k} \omega_{k} b_{k}^{\dagger} b_{k}
$$

and the interaction Hamiltonian is replaced by

$$
H_{I}=\sum_{k}\left(g_{k} \sigma_{+} \otimes b_{k}+g_{k}^{*} \sigma_{-} \otimes b_{k}^{\dagger}\right) .
$$

For a Lorentzian spectral density this model corresponds to the damped Jaynes-Cummings model. The time evolution map for this model, considering the special case of an environment in the vacuum state, has been obtained in [35] and can be expressed as

$$
\begin{gathered}
\rho_{11}(t)=\rho_{11}(0)|G(t)|^{2}, \\
\rho_{10}(t)=\rho_{10}(0) G(t), \\
\rho_{01}(t)=\rho_{01}(0) G^{*}(t), \\
\rho_{00}(t)=\rho_{00}(0)+\rho_{11}(0)\left[1-|G(t)|^{2}\right],
\end{gathered}
$$

where $\rho(t)=\Phi^{\mathrm{DVac}}(t) \rho(0)$, since we are considering the damped model with the bath in the vacuum state. The function $G(t)$ is the solution of the equation

$$
\frac{d}{d t} G(t)=-\int_{0}^{t} \mathrm{dt}_{1} f\left(t-t_{1}\right) G\left(t_{1}\right), \quad G(0)=1,
$$


with $f(t)$ the two-point correlation function given by

$$
\begin{aligned}
f\left(t-t_{1}\right) & =e^{i \omega_{0}\left(t-t_{1}\right)}\left\langle 0\left|\sum_{k} g_{k} b_{k} e^{-i \omega_{k} t} \sum_{j} g_{j}^{*} b_{j}^{\dagger} e^{i \omega_{j} t_{1}}\right| 0\right\rangle \\
& =\sum_{k}\left|g_{k}\right|^{2} e^{i\left(\omega_{0}-\omega_{k}\right)\left(t-t_{1}\right)}
\end{aligned}
$$

corresponding to the Fourier transform of the spectral density.

Starting from Eq. (40) and exploiting the same strategy used in Sec. III, one immediately obtains, for the matrix representation of the time-convolutionless generator, the expression

$$
\begin{aligned}
& K_{\mathrm{TCL}}^{\mathrm{DVac}}(t) \\
& =\left(\begin{array}{cccc}
0 & 0 & 0 & 0 \\
0 & \operatorname{Re}\left[\frac{\dot{G}(t)}{G(t)}\right] & \operatorname{Im}\left[\frac{\dot{G}(t)}{G(t)}\right] & 0 \\
0 & -\operatorname{Im}\left[\frac{\dot{G}(t)}{G(t)}\right] & \operatorname{Re}\left[\frac{\dot{G}(t)}{G(t)}\right] & 0 \\
2 \operatorname{Re}\left[\frac{\dot{G}(t)}{G(t)}\right] & 0 & 0 & 2 \operatorname{Re}\left[\frac{\dot{G}(t)}{G(t)}\right]
\end{array}\right),
\end{aligned}
$$

so that the master equation in operator form reads

$$
\begin{aligned}
\mathcal{K}_{\mathrm{TCL}}^{\mathrm{DVac}}(t) \rho= & +i \operatorname{Im}\left[\frac{\dot{G}(t)}{G(t)}\right]\left[\sigma_{+} \sigma_{-}, \rho\right]-2 \operatorname{Re}\left[\frac{\dot{G}(t)}{G(t)}\right] \\
& \times\left[\sigma_{-} \rho \sigma_{+}-\frac{1}{2}\left\{\sigma_{+} \sigma_{-}, \rho\right\}\right],
\end{aligned}
$$

which confirms the result obtained in [2]. One can also determine the expression of the Nakajima-Zwanzig memory kernel, whose Laplace transform is given by

$$
\begin{aligned}
& \hat{K}_{\mathrm{NZ}}^{\mathrm{DVac}}(u) \\
& =\left(\begin{array}{cccc}
0 & 0 & 0 & 0 \\
0 & u-\frac{\hat{G}_{R}(u)}{\hat{G}_{R^{2}(u)+\hat{G}_{I}{ }^{2}(u)}} & \frac{\hat{G}_{I}(u)}{\hat{G}_{R^{2}(u)+\hat{G}_{I}{ }^{2}(u)}} & 0 \\
0 & -\frac{\hat{G}_{I}(u)}{\hat{G}_{R}{ }^{2}(u)+\hat{G}_{I}{ }^{2}(u)} & u-\frac{\hat{G}_{R}(u)}{\hat{G}_{R}{ }^{2}(u)+\hat{G}_{I}{ }^{2}(u)} & 0 \\
\frac{u \hat{z}(u)-1}{\hat{z}(u)} & 0 & 0 & \frac{u \hat{z}(u)-1}{\hat{z}(u)}
\end{array}\right),
\end{aligned}
$$

where we have used the notation

$$
z(t)=|G(t)|^{2},
$$

leading to the master equation

$$
\begin{aligned}
\widehat{\mathcal{K}}_{\mathrm{NZ}}^{\mathrm{Vac}}(u) \rho= & +i \frac{\hat{G}_{I}(u)}{\hat{G}_{R}^{2}(u)+\hat{G}_{I}^{2}(u)}\left[\sigma_{+} \sigma_{-}, \rho\right] \\
& +\left[\frac{1-u \hat{z}(u)}{\hat{z}(u)}\right]\left[\sigma_{-} \rho \sigma_{+}-\frac{1}{2}\left\{\sigma_{+} \sigma_{-}, \rho\right\}\right] \\
& -\frac{1}{4}\left[\frac{1-u \hat{z}(u)}{\hat{z}(u)}+2\left(u-\frac{\hat{G}_{R}(u)}{\hat{G}_{R}^{2}(u)+\hat{G}_{I}^{2}(u)}\right)\right] \\
& \times\left[\sigma_{z} \rho \sigma_{z}-\rho\right] .
\end{aligned}
$$

\section{B. Role of the correlation function}

We now show that this constructive approach leads to the same result as obtained by a suitable ansatz in [20], where the detailed connection with the perturbative expansion via projection operator techniques was also considered. We first have to recall the relation in Laplace transform between the function $G(t)$ and the function $f(t)$. Indeed, for the case of a two-level system interacting with an environment of oscillators in the vacuum state, all functions appearing in the non-Markovian equations of motion can be related to a single correlation function of the model, given by Eq. (42).

To fully exploit this fact in order to express the memory kernel in the most compact and transparent way, let us observe that, according to Eq. (41), one has

$$
\hat{f}(u)=\frac{\hat{G}^{*}(u)}{|\hat{G}(u)|^{2}}-u,
$$

so that in view of the fact that, for real $u$,

$$
\operatorname{Re}[\hat{h}(u)]=\widehat{\operatorname{Re}} h(u)
$$

together with

$$
\operatorname{Im}[\hat{h}(u)]=\widehat{\operatorname{Im}} h(u),
$$

the functions

$$
\hat{f}_{I}(u) \text { and }-\frac{\hat{G}_{I}(u)}{\hat{G}_{R}^{2}(u)+\hat{G}_{I}^{2}(u)}
$$

do coincide on the real axis and, therefore, due to the identity principle, in the common region of analyticity. A corresponding result holds for the functions

$$
\hat{f}_{R}(u) \text { and } \frac{\hat{G}_{R}(u)}{\hat{G}_{R}^{2}(u)+\hat{G}_{I}^{2}(u)}-u,
$$

so that the matrix representing the Nakajima-Zwanzig kernel can be rewritten in a more compact way as

$$
\hat{K}_{\mathrm{NZ}}^{\mathrm{DVac}}(u)=\left(\begin{array}{cccc}
0 & 0 & 0 & 0 \\
0 & -\hat{f}_{R}(u) & -\hat{f}_{I}(u) & 0 \\
0 & \hat{f}_{I}(u) & -\hat{f}_{R}(u) & 0 \\
\frac{u \hat{z}(u)-1}{\hat{z}(u)} & 0 & 0 & \frac{u \hat{z}(u)-1}{\hat{z}(u)}
\end{array}\right),
$$

leading indeed to the integral kernel first obtained in [20],

$$
\begin{aligned}
\mathcal{K}_{\mathrm{NZ}}^{\mathrm{DVac}}(\tau) \rho= & -i f_{I}(\tau)\left[\sigma_{+} \sigma_{-}, \rho\right] \\
& +k_{1}(\tau)\left[\sigma_{-} \rho \sigma_{+}-\frac{1}{2}\left\{\sigma_{+} \sigma_{-}, \rho\right\}\right] \\
& -\frac{1}{4}\left[k_{1}(\tau)-2 f_{R}(\tau)\right]\left[\sigma_{z} \rho \sigma_{z}-\rho\right],
\end{aligned}
$$

where we have set

$$
\hat{k}_{1}(u)=\frac{1-u \hat{z}(u)}{\hat{z}(u)} .
$$

For the case of a single mode the correlation function considered in Eq. (42) explicitly becomes

$$
f^{1}(t)=g^{2} e^{i \Delta t}
$$

where the superscript again stresses the fact that a single mode is considered. The solution of the integrodifferential Eq. (41) is then exactly given by the function $G^{1}(t)$ introduced in Eq. (32). As should be, Eqs. (33) and (35) are obtained from Eqs. (44) and (47) under the replacement $G(t) \rightarrow G^{1}(t)$, which 
corresponds to the special choice of a single-mode bath. At the same time it is clear that the explicit coefficients appearing in Eq. (37) can indeed be expressed using the real and imaginary parts of Eq. (56), as well as the inverse Laplace transform of

$$
\begin{aligned}
\hat{k}_{1}^{1}(u) & =\frac{1-u \hat{z}^{1}(u)}{\hat{z}^{1}(u)} \\
& =2 g^{2} \frac{u}{u^{2}+\Delta^{2}+2 g^{2}},
\end{aligned}
$$

which is given by

$$
k_{1}^{1}(\tau)=2 g^{2} \cos \left(\sqrt{\Delta^{2}+2 g^{2}} \tau\right) .
$$

\section{Additional term for the thermal bath}

It is to be stressed that the possibility of expressing all relevant functions appearing in time-convolutionless and Nakajima-Zwanzig master equations with reference to the single correlation function $f(t)$ is a special feature of the two-level system coupled to the vacuum. Indeed while the results in Sec. III for the vacuum are a special case of the model considered in Sec. IV, the more general situation of a thermal bath can be explicitly considered for the case of the reduced dynamics of the Jaynes-Cummings model, showing that the expectation values of various operators as in Eq. (13) have to be specified to give the exact equations of motion.

In Sec. III we have shown that the time-convolutionless generator has a different operator structure with respect to the Nakajima-Zwanzig memory kernel only for the case of the vacuum, as it appears upon comparing Eqs. (33) and (37), while this is no longer true for a thermal state. This strongly suggests that the asymmetry in the operator structure of Eqs. (44) and (54) is also due to this special choice of the bath state. To check this fact, in the absence of the exact time evolution map, one has to calculate the time-convolutionless master equation relying on the standard perturbative technique [2], considering terms up to fourth order. The necessity to go up to the fourth perturbative order is immediately clear upon looking at the interaction Hamiltonian, Eq. (39), and observing that the dephasing term, as it appears from Eq. (29), involves a quadrilinear contribution in the raising and lowering operators $\sigma_{+}$and $\sigma_{-}$. This task is accomplished in the Appendix, leading to the result

$$
\begin{aligned}
\mathcal{K}_{\mathrm{TCL}}^{D}(t) \rho= & i \operatorname{Im} \gamma_{s}(t)\left[\sigma_{+} \sigma_{-}, \rho\right] \\
& +\gamma_{+}(t)\left[\sigma_{+} \rho \sigma_{-}-\frac{1}{2}\left\{\sigma_{-} \sigma_{+}, \rho\right\}\right] \\
& +\gamma_{-}(t)\left[\sigma_{-} \rho \sigma_{+}-\frac{1}{2}\left\{\sigma_{+} \sigma_{-}, \rho\right\}\right] \\
& +\frac{1}{4} \gamma_{d}(t)\left[\sigma_{z} \rho \sigma_{z}-\rho\right],
\end{aligned}
$$

and the detailed expression of the various coefficients in terms of two- and four-point correlation functions of the system is given in Eq. (A22) in the Appendix.

This result shows that indeed the disappearance of the dephasing term in the time-convolutionless master equation for the vacuum is a very special feature of this choice of bath. Once again, the operator structure of non-Markovian equations of motion is strongly dependent on the details of both the bath and the interaction terms.

\section{CONCLUSIONS}

In this article we have obtained the exact timeconvolutionless, time-local master equation and NakajimaZwanzig integrodifferential master equation for a two-level system coupled to a single bosonic mode, considering a generic bath state. This has been possible, thanks to the knowledge of the exact dynamics, and corresponds to the result which can be obtained by resumming all terms in the corresponding perturbative techniques. The path followed here-to associate matrices with maps and to consider the direct relation between the time evolution map and the time-local generator, as well as the memory kernel-is, however, much more straightforward. The result shows that, in a realistic model, each operator contribution in Lindblad form has its own time-dependent function, responsible for the non-Markovian behavior, so that a simple multiplication or convolution of the Markovian result with a single phenomenologically guessed function will generally not work. Furthermore, the operator structures of the equations of motion in the two cases can differ greatly, also depending on the state of the bath. In particular, it has been shown that a dephasing term, which is always present in the Nakajima-Zwanzig equations of motion, disappears for the time-convolutionless case for a bath in the vacuum state. This has been checked for the undamped two-level system, thanks to the exact solution, and for the damped case by calculating the fourth-order contribution to the time-convolutionless perturbation expansion.

We would like to stress that, at variance with [20], the present approach is constructive and model independent, not relying on a tentative ansatz. Indeed it has allowed us to cope with a general bath initial state, which, in turn, leads to the appearance of various environmental correlation functions, rather than a single one. In particular, we have shown that the lack of the dephasing term in the time-convolutionless generator of the two-level system dynamics is a peculiarity of the vacuum state, while in [20] it was conjectured that this was a special feature of the time-convolutionless generator, without showing the dependence of this fact on the choice of environmental state. This is an explicit example of how the choice of the initial state for the environment influences the operator structure of the non-Markovian master equation. Note that, at variance with the disappearence of the gain term, this result cannot be guessed on the basis of physical intuition only. Still, at variance with the Markovian case, the presence of each operator term is crucial for preserving complete positivity of the time evolution.

The approach pursued is quite straightforward, even though, to obtain the exact expressions, it relies on the knowledge of the full time evolution, which obviously is feasible only for exceptional cases. The detailed analysis of such cases, however, proves quite useful in understanding the basic features of a non-Markovian description; in particular, it puts into evidence the strict relationship between the different quantities which appear in the evolution equations, showing that phenomenological ansatze are in general not easily feasible. Furthermore, the connection to the microscopic perturbative derivation techniques, as outlined here, can help in determining the operator structure of the dynamical equations. This structure can sometimes be unveiled calculating the first perturbative contributions, thus restricting the number 
of ansatze necessary to consider a sound phenomenological model.

Non-Markovian effects arise because of the formation of non-negligible correlations between a system and its surrounding environment, typically arising in the presence of strong coupling. These correlations in turn imply that a factorized state cannot always be realized, so that the study of the dynamics of correlated initial states becomes of great significance. This long-standing topic has recently seen important advancements [36-43], and we plan to address it in future research work, looking at simple but realistic models, such as the one considered in this article, for general signatures of the effect of initial correlations. In the case of correlated initial states in general systems, however, one can no longer obtain a closed evolution for the degrees of freedom of the reduced system only, so that to achieve a useful description of the dynamics, one possibly has to enlarge the set of degrees of freedom, partly including information from the environment. This of course would have important consequences on the initial states which are experimentally accessible, since the initial preparation would then also imply a partial measurement of the reservoir.

\section{ACKNOWLEDGMENTS}

The authors are grateful to Heinz-Peter Breuer, Federico Casagrande, and Ludovico Lanz for helpful discussions and reading of the manuscript. This work was partially supported by Ministero dell'Istruzione, dell'Università e della Ricerca (MIUR), under PRIN2008.

\section{APPENDIX}

We consider here the contributions up to fourth order of the time-convolutionless projection operator technique for the damped two-level system considered in Sec. IV. We assume for the environment an equilibrium state $\rho_{E}$, which commutes with the number operator, and we use the standard projection operator

$$
\mathcal{P} w=\operatorname{Tr}_{E}(w) \otimes \rho_{E},
$$

where $w$ denotes a state of system and environment.

The time-convolutionless perturbative expansion can be obtained by repeated action of the projection operator considered previously and of the superoperator

$$
\mathcal{L}(t) w=-i\left[H_{I}(t), w\right],
$$

where $H_{I}(t)$ is the interaction picture expression corresponding to Eq. (39), given by

$$
H_{I}(t)=\sigma_{+}(t) \otimes B(t)+\sigma_{-}(t) \otimes B^{\dagger}(t),
$$

with

$$
\sigma_{ \pm}(t)=e^{ \pm i \omega_{0} t}
$$

and

$$
B(t)=\sum_{k} g_{k} b_{k} e^{-i \omega_{k} t}
$$

The contributions to second and fourth order for the timeconvolutionless generator then read [2]

$$
\mathcal{K}_{2}(t)=\int_{0}^{t} d t_{1} \mathcal{P} \mathcal{L}(t) \mathcal{L}\left(t_{1}\right) \mathcal{P}
$$

and

$$
\begin{aligned}
\mathcal{K}_{4}(t)= & \int_{0}^{t} d t_{1} \int_{0}^{t_{1}} d t_{2} \int_{0}^{t_{2}} d t_{3}\left[\mathcal{P} \mathcal{L}(t) \mathcal{L}\left(t_{1}\right) \mathcal{L}\left(t_{2}\right) \mathcal{L}\left(t_{3}\right) \mathcal{P}\right. \\
& -\mathcal{P} \mathcal{L}(t) \mathcal{L}\left(t_{1}\right) \mathcal{P} \mathcal{L}\left(t_{2}\right) \mathcal{L}\left(t_{3}\right) \mathcal{P} \\
& -\mathcal{P} \mathcal{L}(t) \mathcal{L}\left(t_{2}\right) \mathcal{P} \mathcal{L}\left(t_{1}\right) \mathcal{L}\left(t_{3}\right) \mathcal{P} \\
& \left.-\mathcal{P} \mathcal{L}(t) \mathcal{L}\left(t_{3}\right) \mathcal{P} \mathcal{L}\left(t_{1}\right) \mathcal{L}\left(t_{2}\right) \mathcal{P}\right]
\end{aligned}
$$

respectively. Using these expressions one immediately obtains the operator form of the contributions to the timeconvolutionless master equation for the reduced dynamics according to

$$
\mathcal{K}_{\mathrm{TCL}}^{(2)}(t) \rho=\operatorname{Tr}_{E}\left\{\int_{0}^{t} d t_{1} \mathcal{L}(t) \mathcal{L}\left(t_{1}\right) \rho \otimes \rho_{E}\right\}
$$

and

$$
\begin{aligned}
\mathcal{K}_{\mathrm{TCL}}^{(4)}(t) \rho= & \operatorname{Tr}_{E}\left\{\int_{0}^{t} d t_{1} \int_{0}^{t_{1}} d t_{2} \int_{0}^{t_{2}} d t_{3}\right. \\
& \times\left[\mathcal{P} \mathcal{L}(t) \mathcal{L}\left(t_{1}\right) \mathcal{L}\left(t_{2}\right) \mathcal{L}\left(t_{3}\right) \mathcal{P} \rho \otimes \rho_{E}\right. \\
& -\mathcal{P} \mathcal{L}(t) \mathcal{L}\left(t_{1}\right) \mathcal{P} \mathcal{L}\left(t_{2}\right) \mathcal{L}\left(t_{3}\right) \mathcal{P} \rho \otimes \rho_{E} \\
& -\mathcal{P} \mathcal{L}(t) \mathcal{L}\left(t_{2}\right) \mathcal{P} \mathcal{L}\left(t_{1}\right) \mathcal{L}\left(t_{3}\right) \mathcal{P} \rho \otimes \rho_{E} \\
& \left.\left.-\mathcal{P} \mathcal{L}(t) \mathcal{L}\left(t_{3}\right) \mathcal{P} \mathcal{L}\left(t_{1}\right) \mathcal{L}\left(t_{2}\right) \mathcal{P} \rho \otimes \rho_{E}\right]\right\} .
\end{aligned}
$$

We recall that such high-order contributions are needed to check the appearance, for an environmental state different from the vacuum, of the dephasing term $\sigma_{z} \rho \sigma_{z}-\rho$, which involves expressions with altogether four raising and lowering operators of the two-level system.

The second-order contribution Eq. (A8) can be expressed by means of the following two correlation functions:

$$
\begin{aligned}
f\left(t-t_{1}\right) & =e^{i \omega_{0}\left(t-t_{1}\right)} \operatorname{Tr}_{E}\left\{B(t) B^{\dagger}\left(t_{1}\right) \rho_{E}\right\} \\
& =\sum_{k}\left|g_{k}\right|^{2} e^{i\left(\omega_{0}-\omega_{k}\right)\left(t-t_{1}\right)}\left\langle n_{k}+1\right\rangle_{E},
\end{aligned}
$$

which corresponds to Eq. (42) if the bath is in the vacuum state, and

$$
\begin{aligned}
g\left(t-t_{1}\right) & =e^{-i \omega_{0}\left(t-t_{1}\right)} \operatorname{Tr}_{E}\left\{B^{\dagger}(t) B\left(t_{1}\right) \rho_{E}\right\} \\
& =\sum_{k}\left|g_{k}\right|^{2} e^{-i\left(\omega_{0}-\omega_{k}\right)\left(t-t_{1}\right)}\left\langle n_{k}\right\rangle_{E},
\end{aligned}
$$

which vanishes in the vacuum. In terms of these functions, one has

$$
\begin{aligned}
\mathcal{P} \mathcal{L}\left(t_{\alpha}\right) \mathcal{L}\left(t_{\beta}\right) \mathcal{P} \rho \otimes \rho_{E} \\
=-\left[f\left(t_{\alpha}-t_{\beta}\right) \sigma_{+} \sigma_{-} \rho+f^{*}\left(t_{\alpha}-t_{\beta}\right) \rho \sigma_{+} \sigma_{-}\right. \\
\quad+g\left(t_{\alpha}-t_{\beta}\right) \sigma_{-} \sigma_{+} \rho+g^{*}\left(t_{\alpha}-t_{\beta}\right) \rho \sigma_{-} \sigma_{+} \\
\left.\quad-2 \operatorname{Re} f\left(t_{\alpha}-t_{\beta}\right) \sigma_{-} \rho \sigma_{+}-2 \operatorname{Re} g\left(t_{\alpha}-t_{\beta}\right) \sigma_{+} \rho \sigma_{-}\right] \otimes \rho_{E}
\end{aligned}
$$

This result is sufficient to obtain the time-convolutionless master equation up to second order; indeed upon inserting 
Eq. (A12) into Eq. (A8), one obtains

$$
\begin{aligned}
\mathcal{K}_{\mathrm{TCL}}^{(2)}(t) \rho= & -i\left[\mathfrak{f}_{I}(t)+\mathfrak{g}_{I}(t)\right]\left[\sigma_{+} \sigma_{-}, \rho\right] \\
& +2 \mathfrak{f}_{R}(t)\left[\sigma_{+} \rho \sigma_{-}-\frac{1}{2}\left\{\sigma_{-} \sigma_{+}, \rho\right\}\right] \\
& +2 \mathfrak{g}_{R}(t)\left[\sigma_{-} \rho \sigma_{+}-\frac{1}{2}\left\{\sigma_{+} \sigma_{-}, \rho\right\}\right],
\end{aligned}
$$

where we have set

$$
\mathfrak{f}(t)=\int_{0}^{t} d t_{1} f\left(t-t_{1}\right)
$$

and

$$
\mathfrak{g}(t)=\int_{0}^{t} d t_{1} g\left(t-t_{1}\right)
$$

denoting, as usual, the real and imaginary parts with the subscripts $R$ and $I$, respectively.

To consider the fourth-order contribution one has to evaluate the four terms given in Eq. (A9). The last three terms on the right-hand side can be obtained by applying twice the result of Eq. (A12), thus obtaining

$$
\begin{aligned}
\mathcal{P} \mathcal{L}(t) & \mathcal{L}\left(t_{\alpha}\right) \mathcal{P} \mathcal{L}\left(t_{\beta}\right) \mathcal{L}\left(t_{\gamma}\right) \mathcal{P} \rho \otimes \rho_{E} \\
= & {\left[-4 \sigma_{+} \rho \sigma_{-}\left\{\operatorname{Re} f\left(t-t_{\alpha}\right) \operatorname{Re} g\left(t_{\beta}-t_{\gamma}\right)\right.\right.} \\
& \left.+\operatorname{Re} g\left(t-t_{\alpha}\right) \operatorname{Re} g\left(t_{\beta}-t_{\gamma}\right)\right\} \\
& -4 \sigma_{-} \rho \sigma_{+}\left\{\operatorname{Re} g\left(t-t_{\alpha}\right) \operatorname{Re} f\left(t_{\beta}-t_{\gamma}\right)\right. \\
& \left.+\operatorname{Re} f\left(t-t_{\alpha}\right) \operatorname{Re} f\left(t_{\beta}-t_{\gamma}\right)\right\} \\
& +\sigma_{+} \sigma_{-} \rho f\left(t-t_{\alpha}\right) f\left(t_{\beta}-t_{\gamma}\right) \\
& +\rho \sigma_{+} \sigma_{-} f^{*}\left(t-t_{\alpha}\right) f^{*}\left(t_{\beta}-t_{\gamma}\right) \\
& +\sigma_{-} \sigma_{+} \rho g\left(t-t_{\alpha}\right) g\left(t_{\beta}-t_{\gamma}\right) \\
& +\rho \sigma_{-} \sigma_{+} g^{*}\left(t-t_{\alpha}\right) g^{*}\left(t_{\beta}-t_{\gamma}\right) \\
& +\sigma_{+} \sigma_{-} \rho \sigma_{+} \sigma_{-}\left\{2 \operatorname{Re}\left[f\left(t-t_{\alpha}\right) f^{*}\left(t_{\beta}-t_{\gamma}\right)\right]\right. \\
& \left.+4 \operatorname{Re} g\left(t-t_{\alpha}\right) \operatorname{Re} f\left(t_{\beta}-t_{\gamma}\right)\right\} \\
& +\sigma_{-} \sigma_{+} \rho \sigma_{-} \sigma_{+}\left\{2 \operatorname{Re}\left[g\left(t-t_{\alpha}\right) g^{*}\left(t_{\beta}-t_{\gamma}\right)\right]\right. \\
& \left.+4 \operatorname{Re} f\left(t-t_{\alpha}\right) \operatorname{Re} g\left(t_{\beta}-t_{\gamma}\right)\right\} \\
& +\sigma_{-} \sigma_{+} \rho \sigma_{+} \sigma_{-}\left\{f^{*}\left(t-t_{\alpha}\right) g\left(t_{\beta}-t_{\gamma}\right)\right. \\
& \left.+g\left(t-t_{\alpha}\right) f^{*}\left(t_{\beta}-t_{\gamma}\right)\right\} \\
& +\sigma_{+} \sigma_{-} \rho \sigma_{-} \sigma_{+}\left\{g^{*}\left(t-t_{\alpha}\right) f\left(t_{\beta}-t_{\gamma}\right)\right. \\
& \left.+f\left(t-t_{\alpha}\right) g^{*}\left(t_{\beta}-t_{\gamma}\right)\right\} \otimes \rho_{E},
\end{aligned}
$$

where the relations $\sigma_{+}^{2}=\sigma_{-}^{2}=0$ have been repeatedly used, together with the assumption $\left[\rho_{E}, n_{k}\right]=0$.

The first term on the right-hand side of Eq. (A9) instead requires the introduction of a four-point correlation function, which is given by

$$
\begin{aligned}
& h\left(t_{a}, t_{b}, t_{c}, t_{d}\right) \\
& \quad=e^{i \omega_{0}\left(t_{a}-t_{b}+t_{c}-t_{d}\right)} \operatorname{Tr}_{E}\left\{B\left(t_{a}\right) B^{\dagger}\left(t_{b}\right) B\left(t_{c}\right) B^{\dagger}\left(t_{d}\right) \rho_{E}\right\},
\end{aligned}
$$

with $B(t)$ as in Eq. (A5). An explicit evaluation of $\mathcal{P} \mathcal{L}(t) \mathcal{L}\left(t_{1}\right) \mathcal{L}\left(t_{2}\right) \mathcal{L}\left(t_{3}\right) \mathcal{P} \rho \otimes \rho_{E}$ together with the repeated use of Eq. (A16) then leads to the desired result, which can be obtained with a straightforward, though very lengthy, calculation. The fourth-order contribution reads

$$
\begin{aligned}
\mathcal{K}_{\mathrm{TCL}}^{(4)}(t) \rho= & i\left[\mathfrak{p}_{I}(t)+\mathfrak{r}_{I}(t)+\mathfrak{v}_{I}(t)\right]\left[\sigma_{+} \sigma_{-}, \rho\right] \\
& +\mathfrak{t}(t)\left[\sigma_{+} \rho \sigma_{-}-\frac{1}{2}\left\{\sigma_{-} \sigma_{+}, \rho\right\}\right] \\
& +\mathfrak{u}(t)\left[\sigma_{-} \rho \sigma_{+}-\frac{1}{2}\left\{\sigma_{+} \sigma_{-}, \rho\right\}\right] \\
& +\frac{1}{4}\left[\mathfrak{q}(t)+\mathfrak{s}(t)+2 \mathfrak{v}_{R}(t)\right]\left[\sigma_{z} \rho \sigma_{z}-\rho\right],
\end{aligned}
$$

where, in analogy with the notation of Eqs. (A14) and (A15), we use the Fraktur character to denote the triple integral over time of the function with the corresponding Roman letter; for example,

$$
\mathfrak{p}(t)=\int_{0}^{t} d t_{1} \int_{0}^{t_{1}} d t_{2} \int_{0}^{t_{2}} d t_{3} p\left(t, t_{1}, t_{2}, t_{3}\right) .
$$

The functions determining the coefficients appearing in Eq. (A18) are given in terms of the already introduced twoand four-point correlation functions of the model according to the expressions

$$
\begin{aligned}
p\left(t, t_{1}, t_{2}, t_{3}\right)= & -\sum_{\alpha \beta \gamma} f\left(t-t_{\alpha}\right) f\left(t_{\beta}-t_{\gamma}\right)+h\left(t, t_{1}, t_{2}, t_{3}\right) \\
q\left(t, t_{1}, t_{2}, t_{3}\right)= & -2 \sum_{\alpha \beta \gamma}\left\{\operatorname{Re}\left[f\left(t-t_{\alpha}\right) f^{*}\left(t_{\beta}-t_{\gamma}\right)\right]\right. \\
& +2 \operatorname{Re} g\left(t-t_{\alpha}\right) \operatorname{Re} f\left(t_{\beta}-t_{\gamma}\right) \\
& \left.-\operatorname{Re} h\left(t_{\alpha}, t, t_{\beta}, t_{\gamma}\right)\right\} \\
r\left(t, t_{1}, t_{2}, t_{3}\right)= & g\left(t-t_{2}\right) g\left(t_{1}-t_{3}\right)+g\left(t-t_{3}\right) g\left(t_{1}-t_{2}\right) \\
& +f\left(t_{1}-t\right) f\left(t_{3}-t_{2}\right)-h\left(t_{1}, t, t_{3}, t_{2}\right) \\
s\left(t, t_{1}, t_{2}, t_{3}\right)= & -2 \sum_{\alpha \beta \gamma}\left\{\operatorname{Re}\left[f\left(t-t_{\alpha}\right) f\left(t_{\gamma}-t_{\beta}\right)\right]\right. \\
& +2 \operatorname{Re} f\left(t-t_{\alpha}\right) \operatorname{Re} g\left(t_{\beta}-t_{\gamma}\right) \\
& \left.-\operatorname{Re} h\left(t, t_{\alpha}, t_{\gamma}, t_{\beta}\right)\right\} \\
t\left(t, t_{1}, t_{2}, t_{3}\right)= & 2 \sum_{\alpha \beta \gamma}\left\{\operatorname{Re}\left[f\left(t-t_{\alpha}\right) f\left(t_{\gamma}-t_{\beta}\right)\right]\right. \\
& +\operatorname{Re}\left[g\left(t-t_{\alpha}\right) g\left(t_{\beta}-t_{\gamma}\right)\right] \\
& +2 \operatorname{Re} f\left(t-t_{\alpha}\right) \operatorname{Re} g\left(t_{\beta}-t_{\gamma}\right) \\
& \left.-\operatorname{Re} h\left(t, t_{\alpha}, t_{\gamma}, t_{\beta}\right)\right\} \\
& +2\left\{\operatorname{Re}\left[f\left(t_{1}-t\right) f\left(t_{3}-t_{2}\right)\right]\right. \\
& \left.-\operatorname{Re}\left[g\left(t-t_{1}\right) g\left(t_{2}-t_{3}\right)\right]-\operatorname{Re} h\left(t_{1}, t, t_{3}, t_{2}\right)\right\} \\
v\left(t, t_{1}, t_{2}, t_{3}\right)= & 2 \sum_{\alpha \beta \gamma}\left\{f\left(t_{\alpha}-t\right) f\left(t_{\gamma}-t_{\beta}\right)-h\left(t_{\alpha}, t, t_{\gamma}, t_{\beta}\right)\right\}, \\
& \left.-\operatorname{Re} h\left(t_{\alpha}, t, t_{\beta}, t_{\gamma}\right)\right\} \\
u\left(t, t_{1}, t_{2}, t_{3}\right)= & 2 \sum_{\alpha \beta \gamma}\left\{\operatorname{Re} f\left(t-t_{\alpha}\right) \operatorname{Re} f\left(t_{\beta}-t_{\gamma}\right)\right. \\
& +2 \operatorname{Re} g\left(t-t_{\alpha}\right) \operatorname{Re} f\left(t_{\beta}-t_{\gamma}\right) \\
& -\operatorname{Re} h\left(t, t_{1}, t_{2}, t_{3}\right) \\
&
\end{aligned}
$$


where the following summation convention has been used:

$$
\sum_{\alpha \beta \gamma} \psi\left(t_{\alpha}, t_{\beta}, t_{\gamma}\right)=\psi\left(t_{1}, t_{2}, t_{3}\right)+\psi\left(t_{2}, t_{1}, t_{3}\right)+\psi\left(t_{3}, t_{1}, t_{2}\right) .
$$

Including terms up to fourth order, one therefore has the expression Eq. (59) with time-dependent coefficients given by the identifications

$$
\begin{gathered}
\gamma_{s}(t)=-\mathfrak{f}(t)-\mathfrak{g}(t)+\mathfrak{p}(t)+\mathfrak{r}(t)+\mathfrak{v}(t), \\
\gamma_{+}(t)=2 \mathfrak{f}_{R}(t)+\mathfrak{t}(t), \\
\gamma_{+}(t)=2 \mathfrak{g}_{R}(t)+\mathfrak{u}(t), \\
\gamma_{d}(t)=\mathfrak{q}(t)+\mathfrak{s}(t)+2 \mathfrak{v}_{R}(t) .
\end{gathered}
$$

[1] R. Alicki and K. Lendi, Quantum Dynamical Semigroups and Applications, Lecture Notes in Physics, vol. 717, 2nd ed. (Springer, Berlin, 2007).

[2] H.-P. Breuer and F. Petruccione, The Theory of Open Quantum Systems (Oxford University Press, Oxford, 2007).

[3] U. Weiss, Quantum Dissipative Systems, 3rd ed. (World Scientific, Singapore, 2008).

[4] A. A. Budini, Phys. Rev. A 69, 042107 (2004).

[5] A. A. Budini, Phys. Rev. E 72, 056106 (2005).

[6] A. A. Budini and H. Schomerus, J. Phys. A 38, 9251 (2005).

[7] A. A. Budini, Phys. Rev. A 74, 053815 (2006).

[8] S. Maniscalco and F. Petruccione, Phys. Rev. A 73, 012111 (2006).

[9] H.-P. Breuer, J. Gemmer, and M. Michel, Phys. Rev. E 73, 016139 (2006).

[10] H.-P. Breuer, Phys. Rev. A 75, 022103 (2007).

[11] B. Vacchini, Phys. Rev. A 78, 022112 (2008).

[12] E. Ferraro, H.-P. Breuer, A. Napoli, M. A. Jivulescu, and A. Messina, Phys. Rev. B 78, 064309 (2008).

[13] A. Kossakowski and R. Rebolledo, Open Syst. Inf. Dyn. 15, 135 (2008).

[14] A. Kossakowski and R. Rebolledo, Open Syst. Inf. Dyn. 16, 259 (2009).

[15] H.-P. Breuer and B. Vacchini, Phys. Rev. Lett. 101, 140402 (2008).

[16] H.-P. Breuer and B. Vacchini, Phys. Rev. E 79, 041147 (2009).

[17] J. Piilo, S. Maniscalco, K. Harkonen, and K.-A. Suominen, Phys. Rev. Lett. 100, 180402 (2008).

[18] J. Piilo, K. Härkönen, S. Maniscalco, and K.-A. Suominen, Phys. Rev. A 79, 062112 (2009).

[19] H.-P. Breuer and J. Piilo, Europhys. Lett. 85, 50004 (2009).

[20] B. Vacchini and H.-P. Breuer, Phys. Rev. A 81, 042103 (2010).

[21] D. Chruscinski and A. Kossakowski, Phys. Rev. Lett. 104, 070406 (2010).
[22] D. Chruscinski, A. Kossakowski, and S. Pascazio, Phys. Rev. A 81, 032101 (2010).

[23] E.-M. Laine, J. Piilo, and H.-P. Breuer, Phys. Rev. A 81, 062115 (2010).

[24] L. Mazzola, E.-M. Laine, H.-P. Breuer, S. Maniscalco, and J. Piilo, Phys. Rev. A 81, 062120 (2010).

[25] V. Gorini, A. Kossakowski, and E. C. G. Sudarshan, J. Math. Phys. 17, 821 (1976).

[26] G. Lindblad, Commun. Math. Phys. 48, 119 (1976).

[27] R. R. Puri, Mathematical Methods of Quantum Optics (Springer, Berlin, 2001).

[28] E. Andersson, J. D. Cresser, and M. J. W. Hall, J. Mod. Opt. 54, 1695 (2007).

[29] J. Salo, S. M. Barnett, and S. Stenholm, Opt. Commun. 259, 772 (2006).

[30] J. Cresser and C. Facer, Opt. Commun. 283, 773 (2010).

[31] S. M. Barnett and S. Stenholm, Phys. Rev. A 64, 033808 (2001).

[32] A. Shabani and D. A. Lidar, Phys. Rev. A 71, 020101 (2005).

[33] S. Maniscalco, Phys. Rev. A 75, 062103 (2007).

[34] J. Wilkie and Y. M. Wong, J. Phys. A 42, 015006 (2009).

[35] H.-P. Breuer, B. Kappler, and F. Petruccione, Phys. Rev. A 59, 1633 (1999).

[36] P. Pechukas, Phys. Rev. Lett. 73, 1060 (1994).

[37] R. Alicki, Phys. Rev. Lett. 75, 3020 (1995).

[38] A. Royer, Phys. Rev. Lett. 77, 3272 (1996).

[39] A. J. van Wonderen and K. Lendi, J. Phys. A 39, 14511 (2006).

[40] A. Shabani and D. A. Lidar, Phys. Rev. Lett. 102, 100402 (2009).

[41] C. A. Rodríguez-Rosario, K. Modi, and A. Aspuru-Guzik, Phys. Rev. A 81, 012313 (2010).

[42] P. Aniello, A. Kossakowski, G. Marmo, F. Ventriglia, and P. Vitale, Open Syst. Inf. Dyn. 17, 21 (2010).

[43] E.-M. Laine, J. Piilo, and H.-P. Breuer, e-print arXiv:1004.2184v1. 